\title{
Evaluation of Appropriateness of Antibiotic Use and Validation of the Mclsaac-Modified Centor Score for Group A Beta Hemolytic Streptococcal Acute Pharyngitis in Suez Canal Area
}

\author{
Mohamed F. Hassan', Mohamed M. Eida', Lobna A. Metwally², Mahmoud H. \\ Ahmed $^{1^{*}}$
}

Departments of ${ }^{1}$ Endemic and Infectious diseases and ${ }^{2}$ Microbiology and Immunology, Faculty of Medicine, Suez Canal University, Egypt

\begin{abstract}
Background: Acute pharyngitis is one of the most common illnesses. Most acute cases are caused by viral infections. To enhance the appropriate prescribing of antibiotics a number of clinical prediction rules have been developed to distinguish streptococcal pharyngitis from pharyngitis by other causes Aim: To assess the appropriateness of antibiotic prescribed to cases of acute pharyngitis and the diagnostic accuracy of the Mclsaac modified Centor score for predicting group A beta hemolytic streptococcal pharyngitis. Patients and Methods: A cross-sectional study; was conducted in Port Said fever hospital, Ismailia fever hospital and Suez fever hospital, within the area of Suez Canal, including. The study included 196 patients who presented to these hospitals between the age of 3 and 65 years. Results: Antibiotics were prescribed to $86 \%$ of patients while the prevalence of group A beta hemolytic streptococcus (GABHS) pharyngitis was 12.8\%. A broad spectrum third-line agents or a non recommended antibiotics were prescribed to $62 \%$ of patients. Mclsaac score was found to be $100 \%$ sensitive and $63.16 \%$ specific giving $28.41 \%$ a positive predictive value and a negative predictive value of $100 \%$. Conclusion: Antibiotics are prescribed inappropriatly to patients with acute pharyngitis and Mclsaac score is a useful clinical to for diagnosis of GABHS pharyngitis and its use decreases antibiotic prescription.
\end{abstract}

Key words: GABHS, cuture, throat

\section{Introduction}

Acute pharyngitis is one of the most common illnesses for which patients visit primary care physicians. Pharyngitis is the inflammation of the pharynx, hypopharynx, tonsils and uvula. Sore throat, fever, and malaise associated with acute pharyngitis are distressing, however, with few exceptions, these illnesses are both benign and self-limited ${ }^{(1)}$. Most acute cases are caused by viral infections with the remainder caused by bacterial infections, fungal infections, or irritants such as pollutants or chemical substances $^{(2)}$. Group A beta hemolytic streptococcus (GABHS) is by far the most common bacterial cause of acute pharyngitis, accounting for approximately $15-30 \%$ of cases in children and 5-17 \% of cases in adults. Moreover, it is the only common form of the disease for which antimicrobial therapy is definitely indicated ${ }^{(3]}$. 
A properly performed and interpreted throat culture remains the gold standard for the diagnosis of GABHS pharyngitis. It has a sensitivity of 90 percent or higher ${ }^{(4)}$. Obtaining definitive results from the throat culture takes between 24 and 48 hours. Delaying antimicrobial therapy for this period will not diminish its efficacy in preventing rheumatic fever, but it is often difficult to explain to patients the need to withhold therapy ${ }^{(5)}$. These problems may eventually be obviated by the rapid antigen-detection test (RADT), which can confirm the presence of GABHS carbohydrate antigen on a throat swab in a matter of minutes. The sensitivity of most of these tests ranges, at best, between $80-90 \%$ when the tests are compared with the blood agar plate culture $^{(4)}$. Neither the conventional throat culture nor the rapid test reliably differentiates acutely infected patients from asymptomatic carriers with intercurrent viral pharyngitis ${ }^{(6)}$. Because antibiotic treatment should occur fairly promptly, diagnosis of pharyngitis is often based on clinical symptoms ${ }^{(7)}$. To enhance the appropriate prescribing of antibiotics without performing cultures to all patients, a number of clinical prediction rules (CPRs) have been developed to distinguish streptococcal pharyngitis from pharyngitis by other causes ${ }^{(8)}$. The Centor criteria are reliable predictors of GABHS pharyngitis. They include evaluating patients for tonsillar exudates, tender anterior cervical lymphadenopathy or lymphadenitis, absence of cough, and history of fever (oral temperature greater than $38.3^{\circ} \mathrm{C}$ ). Patients receive a point for the presence or absence of signs and symptoms. Each patient is assigned a score between 0 and $4^{(9)}$. The Mclsaac score adjusts the Centor score for the patient's age. Since younger patients are more likely to have GABHS than older patients, the Mclsaac score is calculated by adding 1 point to the Centor score for patients aged
3 to 14 years and subtracting 1 point for those 45 years or older. The use of the Mclsaac Modified Centor score has helped in decreasing inappropriate antibiotic use by almost $88 \%^{(8-10)}$. Inappropriate prescribing of antibiotics has been attributed to a number of causes, including people who insist on antibiotics, physicians who simply prescribe them as they feel they do not have time to explain why they are not necessary, and physicians who do not know when to prescribe antibiotics or else are overly cautious for medical legal reasons ${ }^{(11)}$. Given the impact of the antibiotic abuse and overuse and antibiotic resistance and not knowing how physicians generally treat their adult pharyngitis patients, the study investigated whether antibiotics are prescribed appropriately for primary treatment of pharyngitis. The study also evaluated the sensitivity and specificity of Mclsaac Modified Centor scores in predicting GABHS pharyngitis in our population.

\section{Patients and Methods}

A cross-sectional study that was conducted in three Suez Canal area fever hospitals; Port Said, Ismailia and Suez fever hospitals, including the outpatient clinics and the emergency rooms. The study included 196 patients who presented to Suez Canal fever hospitals between the age of 3 and 65 years, after taking their ethical approval. Inclusion criteria: Patients with signs and symptoms suggestive of pharyngitis, patients aged between 3 to 65 years and both genders. Exclusion criteria: Receipt of antibiotics 24-48 hours before symptoms appear, Immunocompromised (i.e. including HIV/AIDS or were on immunosuppressive agents or cytotoxic therapy), Organ transplantation, history of malignancy, evidence of chronic liver disease, end stage kidney disease, diabetes mellitus and serious cardiovascular disease. Patients were inter- 
viewed and evaluated in a separate room after being seen by the hospital's physician in order to determine whether or not the action taken by the physician was consistent with the recommended action according to the Mclsaac clinical rule.

\section{Results}

The study included 196 patients of both genders aged 3 to 65 years who presented to Suez Canal Fever Hospitals with a diagnosis of acute pharyngitis (male, $57.7 \%$, females, $42.3 \%$, and mean age, 21 years) [Table 1]. Most of the patients were among the age group 3-14 years accounting for $46.43 \%$, while the age group $45-65$ years accounted for $14.78 \%$. The remaining $39.8 \%$ of patients were among the age group 1544 years [Table 2]. Sore throat was the most common presenting symptom accounting for $56.6 \%$, while fever and cervical swelling accounted for $29.08 \%$ and $14.29 \%$ respectively of the patients' presenting symptoms [Table 3].

Table 1: Patient demographics

\begin{tabular}{|l|c|}
\hline Variables & Frequency \\
\hline Patients' total number & 196 \\
Males, N (\%) & $133(57.65 \%)$ \\
Females, N (\%) & $83(42.35 \%)$ \\
Mean age (yrs) \pm SD & $21.03 \pm 15.82$ \\
\hline
\end{tabular}

Table 2: Age groups frequencies

\begin{tabular}{|l|cc|}
\hline Age group (Yrs) & Frequency & $\%$ \\
\hline $3-14$ & 91 & $46.43 \%$ \\
$15-44$ & 78 & $39.8 \%$ \\
$45-65$ & 27 & $14.78 \%$ \\
Total & 196 & $100 \%$ \\
\hline
\end{tabular}

Table 3: The patients' main complaint

\begin{tabular}{|l|cc|}
\hline Complaint & Number $(\%)$ & $95 \%(\mathrm{Cl})$ \\
\hline Sore throat & $111(56.6 \%)$ & $(49.3-63.6)$ \\
Fever & $57(29.0 \%)$ & $(22.8-35.9)$ \\
Cervical & $28(14.2 \%)$ & $(9.2-19.9)$ \\
swelling & & \\
\hline
\end{tabular}

$\mathrm{Cl}=$ Confidence Interval, $n=196$
Thirty-six patients (scores 0, 1, 2 and 3), 28 patients (score 4), and 24 patients (score 5) were evaluated for bacterial pharyngitis $(\mathrm{N}=196)$; of these patients, only $25(12.76 \%$, $95 \% \mathrm{Cl} 8.43 \%$ to $18.25 \%$ ) were positive for GABHS as confirmed by throat culture, however, 169 (86.22\%, 95\% Cl 80.59\% to $90.72 \%$ ) were treated with antibiotics. Antibiotics were prescribed inappropriately to patients with pharyngitis; no association was found between antibiotic use and culture results [Table 4]. There was also no association between antibiotic prescription and the results of Mclsaac modified Centor score system. Patients in score groups 0, 1 and 2 represented 108/196 (55.1\%). Of them, $81(75 \%)$ received antibiotics, accounting for 81 out of 169 patients (47.93\%) who received antibiotic prescription. On the other hand, patients in score groups 3, 4 and 5 represented $88 / 96$ (44.9\%) and all of them were prescribed antibiotics (100\%), accounting for $52.07 \%$ of all antibiotic prescriptions [Table 5]. As few as 30/169 (17.75\%) of the patients received first-line agents for treatment of pharyngitis and 34 (20.12\%) of patients received second-line agents while most of the patients 105 (62.13\%) received inappropriate broadspectrum third-line agents or an antibiotic not recommended for GABHS. Of the inappropriate antibiotics prescribed, broadspectrum penicillin $42.60 \%$ were prescribed most followed by macrolides (prescribed in $15.98 \%$ ), including azithromycin, erythromycin for non-penicillin allergic patients and clarithromycin. Cefotaxim, the third generation cephalosporin, was prescribed for $2.37 \%$ patients and the fluoroquinolone, ciprofloxacin were prescribed in $1.18 \%$ [Table 6]. A cross tabulation of Mclsaac modification of Centor score and throat culture found that the score is $100 \%$ sensitive and $63.16 \%$ specific giving $28.41 \%$ a positive predictive value (PPV) and a negative predictive value (NPV) of 100\% [Table 7]. 


\section{Discussion}

This study was carried out in three Fever Hospitals of Suez Canal area and included 196 patients of both genders aged 3 to 65 years, to evaluate the appropriate use of antibiotics in patients with acute pharyngitis and to verify the use of a clinical score to diagnose GABHS pharyngitis. In this study there were more males presenting with symptoms of acute pharyngitis accounting for $57.65 \%$, than females who were $42.35 \%$. This was in agreement with the findings of Palla et al. ${ }^{(12)}$ in Pakistan who found that $66 \%$ were males and $34 \%$ were females; however this was against what was found by Nakhoul and Hickner ${ }^{(13)}$ in USA who found that $69 \%$ of patients were females.

Table 5: Cross tabulation of Mclsaac modification of Centor score and antibiotic prescription

\begin{tabular}{|l|lcc|}
\hline \multirow{2}{*}{$\begin{array}{l}\text { Mclsaac modified } \\
\text { Centor score }\end{array}$} & \multicolumn{3}{|c|}{ Antibiotic prescription } \\
\cline { 2 - 4 } Score 0 & $18(50 \%)$ & $18(50 \%)$ & 36 \\
Score 1 & $27(75 \%)$ & $9(25 \%)$ & 36 \\
Score 2 & $36(100 \%)$ & 0 & 36 \\
Score 3 & $36(100 \%)$ & 0 & 36 \\
Score 4 & $28(100 \%)$ & 0 & 28 \\
Score 5 & $24(100 \%)$ & 0 & 24 \\
\hline Total & $169(86.22 \%)$ & $27(13.78 \%)$ & 196 \\
\hline
\end{tabular}

The mean age of our patients was 21.03 years. The study showed that the most frequently presented patients with symptoms of acute pharyngitis were among the age group 3-14 years accounting for $46.43 \%$, while patients in the age group $15-44$ years accounted for $39.8 \%$ and the age group 45-65 years accounted for $14.78 \%$. The mean age of the patients in the study by Humair et al. ${ }^{(14)}$ was 29.6 years, while Mclsaac et al. ${ }^{(15)}$ in Canada found that the median age of the sample was 16 years with $(57.7 \%)$ aged $3-17$ years and (42.3\%) aged 18-69 years. The study showed that sore throat was the main presenting complaint of the patients accounting for $56.63 \%$. Nandi et al. ${ }^{(16)}$ found that the most common symptom of GABHS pharyngitis was pain in the throat accounting for (86.2\%). Palla et al. $^{(12)}$ reported that the prevalence of GABHS. pharyngitis was $4.38 \%$, in contrast to our study that found the prevalence of GABHS was $12.76 \%$. The difference might be attributed to the fact that Palla and colleagues searched for GABHS pharyngitis in adults only in contrast to our study that included children and adults. However, our prevalence was consistent with the findings of Mclsaac et al. ${ }^{(8)}$ in Canada and Nandi et al. ${ }^{(16)}$ which found that the prevalence of GABHS was $13.8 \%$ and $13.4 \%$ respectively. The study showed that much antibiotic prescription was unnecessary for patients who have acute pharyngitis; probably because most of the patients have viral, rather than bacterial, infection. Actually the study showed that, $12.76 \%$ of patients investigated were found positive for GABHS. Furthermore, the physicians, who prescribed antibiotics, are prescribing broadspectrum agents, such as amoxicillin clavulinic acid or macrolides, instead of using narrow-spectrum agents. Even when the choice of antibiotic was appropriate as recommended by current guidelines ${ }^{(17)}$, 
doses and duration of these agents were mostly inappropriate. The present study found that penicillin group was the most frequently prescribed antibiotic class $60.4 \%$, with broad spectrum penicillin accounting for $42.6 \%$, followed by first generation cephalosporins in $20.1 \%$, macrolides in $16 \%$, third generation cephalo- sporins in $2.4 \%$ and quinolones in $1.2 \%$ of all antibiotic prescriptions. Some of these findings agreed with those of Palla et al..$^{(12)}$ who found that penicillin was the most frequently prescribed (34.1\%), with broad spectrum penicillin accounting for $28.2 \%$ of all prescriptions.

Table 6: Antibiotics prescribed by physicians for pharyngitis patients

\begin{tabular}{|l|lc|}
\hline Appropriate antibiotic Class & Name & No. (\%) \\
\hline First-line agents & Ampicillin & $25(14.79 \%)$ \\
(Penicillins) & Amoxicillin & $5(2.96 \%)$ \\
& Total & $30(17.75 \%)$ \\
Second-line agents & Cephradine & $12(7.10 \%)$ \\
(First generation Cephalosporins) & Cefadroxil & $12(7.10 \%)$ \\
& Cephalexin & $10(5.92 \%)$ \\
Third line (Inappropriate) agents & Total & $34(20.12 \%)$ \\
i. Broad spectrum Penicillins & Amoxicillin clavulinic acid & $37(21.89 \%)$ \\
& Flucloxacillin amoxicillin & $26(15.38 \%)$ \\
& Ampicillin sulbactam & $8(4.73 \%)$ \\
ii. Macrolides & Cloxacillin ampicillin & $1(0.59 \%)$ \\
& & \\
iii. Third generation Cephalosporin & Cefotaxim & $12(7.10 \%)$ \\
iv. Quinolones & Erythromycin & $9(5.33 \%)$ \\
& Clarithromycin & $6(3.55 \%)$ \\
& Ciprofloxacin & $4(2.37 \%)$ \\
& Total & $2(1.18 \%)$ \\
\hline
\end{tabular}

$\$=$ for patient with no history of penicillin allergy, ${ }^{\circledR}=$ Non-recommended antibiotics

Our study showed that $86.22 \%$ of the patients received antibiotic prescription and this even exceeds what was noted by Bisno et al. ${ }^{(18)}$ and reported by Linder and Stafford ${ }^{(19)}$ who found that, nationally antibiotics were prescribed in $73 \%$ of adults with sore throats, a percentage that greatly exceeds the expected prevalence of streptococcal pharyngitis. American College of Physicians and Centers for Disease Control and Prevention guidelines for the Management of Pharyngitis implies that patients with Mclsaac score 0 and 1 should not undergo any testing or receive antibiotics, while patients in score group 2 should be treated if RADT is positive. Patients in score group 3 either to treat empirically or treat if RADT is positive. Patients with score 4 and 5 are treated empirically with antibiotics ${ }^{(20,21)}$. In our study, $62.5 \%$ of patients in score groups 0 and 1 were treated with antibiotics and $100 \%$ of patients in score groups 2, 3, 4 and 5 received antibiotic prescription. It appears that physicians' decisions to prescribe an antibiotic for these patients 
were based solely on their clinical judgment. In this study, broad spectrum antibiotics were prescribed to $62.13 \%$ of patients who received antibiotics. In the
United States, Steinman et al. ${ }^{(22)}$ found that broad spectrum antibiotics were prescribed in more than half of all cases of acute respiratory tract infections in adults.

Table 7: Cross tabulation of Mclsaac modification of Centor score and throat culture

\begin{tabular}{l|cccccc|}
\hline $\begin{array}{l}\text { Mclsaac modification of } \\
\text { Centor score }\end{array}$ & $\begin{array}{c}\text { Positive cul- } \\
\text { ture }\end{array}$ & $\begin{array}{c}\text { Negative cul- } \\
\text { ture }\end{array}$ & Total & TN & TP & FP \\
\hline Score $0(n=36)$ & 0 & $36(100 \%)$ & 36 & 36 & - & 0 \\
Score $1(n=36)$ & 0 & $36(100 \%)$ & 36 & 36 & - & 0 \\
Score 2 $(n=36)$ & 0 & $36(100 \%)$ & 36 & 36 & - & 0 \\
Score 3 $(n=36)$ & $3(8.33 \%)$ & $33(91.67 \%)$ & 36 & 0 & 3 & 33 \\
Score 4 $(n=28)$ & $8(28.57 \%)$ & $20(71.43 \%)$ & 28 & 0 & 8 & 20 \\
Score 5 $(n=24)$ & $14(58.33 \%)$ & $10(41.67 \%)$ & 24 & 0 & 14 & 10 \\
\hline Sensitivity = 100\%, Specificity= 63.16\%, NPV=100\%, PPV= 28.41\% \\
Positive culture= Number of patients found to be GABHS positive in culture/Total number of \\
patients with respective score; Negative culture= Number of patients found to be GABHS \\
negative in culture/Total number of patients with respective score. TN = True negative; TP = \\
True positive; FP = False positive; PPV = positive predictive value; NPV = negative predictive \\
value.
\end{tabular}

According to our study, first line agents for treatment of GABHS as recommended by the current practice guidelines ${ }^{(23)}$ were used only in $17.8 \%$ of patients, who received antibiotics, while second line agents, that are recommended for patients allergic to penicillin $^{(21)}$, were used in $20.1 \%$ of those patients. The rest $62.1 \%$ were prescribed a third line or inappropriate agents. The present study showed that the Mclsaac Modified Centor score system for predicting GABHS pharyngitis was found to be $100 \%$ sensitive and $63.16 \%$ specific giving PPV of $28.41 \%$ and NPV of $100 \%$. Mclsaac and colleagues ${ }^{(15)}$ found that the modified Centor score had a sensitivity of $100 \%$ and specificity of $93.2 \%$ giving PPV of $85.71 \%$ and NPV of $100 \%$. Palla and colleagues ${ }^{(12)}$ in their study to assess the modified Centor score found that it had a sensitivity of $100 \%$ and specificity of $68.7 \%$, giving a PPV of $12.7 \%$ and a NPV of $100 \%$. The study showed that none of the patients in Mclsaac score group 0, 1 and 2 had a positive culture for GABHS, $8.3 \%$ of patients in score group 3, $28.6 \%$ of patients in score group 4 and $58.3 \%$ of patients in score group 5 had positive culture for GABHS. On the other hand, Fine et al. ${ }^{(21)}$ found that $8 \%$ of patients in Mclsaac score o had positive culture result, $14 \%$ of patients in score $1,23 \%$ in score $2,37 \%$ of patients in score 3 and $55 \%$ of patients in score 4 and 5 had positive culture for GABHS. Based on the prescriptions for our patients in score groups 0,1 and 2, if the score-only approach was used, we would save $47.9 \%$ of patients from receiving unnecessary antibiotics. Although without a throat culture or a RADT test option, more antibiotics would be prescribed for patients with a score of 3, 4 and 5 . Actually $100 \%$ of our patients in these score groups received antibiotics accounting for $52.1 \%$ of patients who received antibiotics, but this percentage is better than the percentage of patients getting inappropriate antibiotics without clinical score-based screening at all. It was demonstrated that, the Mclsaac Modified Centor score is very useful for our clinicians as it is not costly, and is sensitive and specific enough to reduce unnecessary antibiotic prescrip-tions. According to our study, if the clinicians used the score-only 
approach instead of depending only on their clinical judgment, we would save antibiotic prescriptions for many of our patients. In addition, Fine et al. ${ }^{(21)}$ stated that they had validated the Centor and Mclsaac scores as useful and valid tools for the diagnosis and treatment of patients with acute pharyngitis.

\section{Conclusion}

Antibiotic treatment of acute pharyngitis benefits only those patients with GABHS infection Antibiotics are prescribed inappropriatly to patients with acute pharyngitis and Mclsaac score is a useful clinical to for diagnosis of GABHS pharyngitis and its use decreases antibiotic prescription. Our study recommends: 1 ). All patients with pharyngitis should be offered appropriate doses of analgesics and antipyretics, as well as other supportive care. 2) Antibiotics should be prescribed only for who are most likely to have GABHS infection as evident by the presence of the Centor criteria; history of fever, tonsillar exudate, no cough, and tender anterior cervical lymphadenopathy. Patients with none or only one of these criteria should not be tested or treated with antibiotics, because these patients are unlikely to have GABHS infection. For patients with two or more criteria the following strategies are appropriate: a) Test patients with two, three, or four criteria by using a rapid antigen test, and limit antibiotic therapy to patients with positive test results. b) Test patients with two or three criteria by using a rapid antigen test or throat culture, and limit antibiotic therapy to patients with positive test results or patients with four criteria. c) Do not use any diagnostic tests, and limit antibiotic therapy to patients with three or four criteria. d) The preferred antibiotic for treatment of acute GABHS phayngitis is penicillin or erythromycin in a penicillin-allergic patient.

\section{References}

1. Cooper RJ, Hoffman JR, Bartlett JG, et al. Principles of appropriate antibiotic use for acute pharyngitis in adults: background. Ann Intern Med. 2001; 134 (6): 509-517.

2. Kontrick AV, Singer JI, Gebhart ME. In Rosen's emergency medicine: concepts and clinical practice (7th Ed) Vol (1); 2010. John MA (Ed). "Chapter 30: Sore Throat".pp: 217. Mosby/Elsevier, Philadelphia, PA.

3. Poses RM, Cebul RD, Collins M, Fager SS. The accuracy of experienced physicians' probability estimates for patients with sore throats: implications for decision making. JAMA. 1985; 254(7): 925 - 929.

4. Bisno AL, Gerber MA, Gwaltney JM, Kaplan EL,Schwartz : Infectious Diseases Society of America. Practice guidelines for the diagnosis and management of group A streptococcal pharyngitis. Infectious Diseases Society of America. Clin Infect Dis. 2002; 35(2): $113-125$.

5. Report of the Committee on Infectious Diseases. Pickering LK, ed. Red book: group A streptococcal infections, 25th ed. Elk Grove Village, Ill.: American Academy of Pediatrics, 2000; 526-536.

6. Nyquist AC, Gonzales R, Steiner JF, Sande MA. Antibiotic prescribing for children with colds, upper respiratory tract infections, and bronchitis. JAMA. 1998; 279 (11): $875-877$.

7. Mclsaac WJ, Goel V. Sore throat practices of Canadian family physicians. Fam Pract. 1997; 14(1): 34-39.

8. Mclsaac WJ, White D, Tannenbaum D, Low DE. A clinical score to reduce unnecessary antibiotic use in patients with sore throat. CMAJ. 1998; 158 (1): 7583.

9. Centor RM, Witherspoon JM, Dalton HP, Brody CE, Link K. The diagnosis of strep throat in adults in the emergency room. Med Decis Making. 1981; 1(3): 239-246.

10. Bisno AL. Diagnosing strep throat in the adult patient: do clinical criteria really 
suffice? Ann Intern Med. 2003; 139(2): 150-151.

11. Arnold SR, Straus SE. Interventions to improve antibiotic prescribing practices in ambulatory care. Cochrane Database Syst Rev 2005;19(4): CDo03539.

12. Palla AH, Khan RA, Gilani AH, Marra F. Over prescription of antibiotics for adult pharyngitis is prevalent in developing countries but can be reduced using Mclsaac modification of Centor scores: a cross-sectional study. BMC Pulm Med. 2012; $12(1): 70$.

13. Nakhoul GN, Hickner J. Management of adults with acute streptococcal pharyngitis: minimal value for backup strep testing and overuse of antibiotics. J Gen Intern Med. 2013; 28(6): 830-834.

14. Humair JP, Revaz SA, Bovier P, Stalder H. Management of acute pharyngitis in adults: reliability of rapid streptococcal tests and clinical findings. Arch Intern Med.2006;166(6): 640-644.

15. Mclsaac WJ, Kellner JD, Aufricht P, Vanjaka A, Low DE. Empirical validation of guidelines for the management of pharyngitis in children and adults. JAMA. 2004; 291(13): 1587-1595.

16. Nandi S, Kumar R, Ray P, Vohra H, Ganguly NK. Clinical score card for diagnosis of group A streptococcal sore throat. Indian J Pediatr. 2002; 69(6): 471475.

17. Shulman ST, Bisno AL, Clegg HW, et al. Clinical practice guideline for the diagnosis and management of group $A$ streptococcal pharyngitis: 2012 update by the Infectious Diseases Society of America. Clin Infect Dis. 2012; 55(10):e86102.

18. Bisno AL, Peter GS, Kaplan EL. Diagnosis of strep throat in adults: are clinical criteria really good enough? Clin Infect Dis. 2002; 35(2): 126-129.

19. Linder JA, Stafford RS. Antibiotic treatment of adults with sore throat by community primary care physicians: a national survey, 1989-1999. JAMA. 2001; 286(10): 1181-1186.

20. Snow V, Mottur-pilson C, Cooper RJ,et al. Principles of appropriate antibiotic use for acute pharyngitis in adults. Ann Intern Med. 2001; 134(6): 506-508.

21. Fine $A M$, Nizet $V$, Mandl KD. Large-scale validation of the Centor and Mclsaac scores to predict group A streptococcal pharyngitis. Arch Intern Med. 2012; 172 (11): 847-852.

22. Steinman MA, Landefeld CS, Gonzales R. Predictors of broad-spectrum antibiotic prescribing for acute respiratory tract infections in adult primary care. JAMA 2003; 289(6): 719-725.

23. Marcy SM. Treatment options for streptococcal pharyngitis. Clinical Pediat-rics (Phila). 2007; 46(suppl 1): 36S-45S. 